\title{
Determinantes para una lactancia materna exitosa: intervención integral vs cuidado estándar. Ensayo clínico aleatorio controlado
}

\author{
CAMILA LUCCHINI R. ${ }^{1}$, CLAUDIA URIBE T. ${ }^{2}$, \\ LUIS VILLARROEL DEL P. ${ }^{3}$, ANTONIA ROJAS R. ${ }^{2}$ \\ 1. Departamento de Salud del Niño y Adolescente, Escuela de Enfermería, Pontificia Universidad Católica de Chile. \\ 2. Departamento de Salud de la Mujer, Escuela de Enfermería, Pontificia Universidad Católica de Chile. \\ 3. Departamento de Salud Pública, Escuela de Medicina, Pontificia Universidad Católica de Chile.
}

\begin{abstract}
Randomized controlled clinical trial evaluating determinants of successful breastfeeding: Follow-up two months after comprehensive intervention versus standard care delivery

Introduction: Exclusive breastfeeding (EBF) is the best alternative for feeding newborns and infants, benefiting the child, the mother, family and society. There are positive and negative covariates influencing the initiation and maintenance of EBF. Objective: To assess the effects of covariates in maintaining EBF after two months, according to type of care received. Patients and Method: Randomized controlled clinical trial with follow-up of women and children regarding prevalence of EBF after 8 weeks, and positive and negative EBF covariates in the two forms of delivery. Sample: 649 postpartum women (330 received comprehensive care and 319 traditional form of care). Results: Prevalence of EBF at 8 weeks corresponded to $56.1 \%$ in comprehensive mode and $48.9 \%$ in traditional mode $(p=0.06)$. There are significantly different EBF results at 8 weeks according to age, birth type and education between both cares. The positive EBF covariates are greater reported for the comprehensive care $(\mathrm{p}<0.0001)$. Conclusion: Although there are no variables that independently affect the EBF initiation and duration at 8 weeks, the comprehensive care benefits them.

(Key words: Exclusive breastfeeding prevalence, comprehensive care delivery).

Rev Chil Pediatr 2013; 84 (2): 138-144
\end{abstract}

\section{RESUMEN}

Introducción: Lactancia materna exclusiva (LME) es la mejor alternativa para alimentar a los recién nacidos y lactantes, reportándose beneficios para el niño(a), la madre, familia y sociedad. Existen "co-variables" positivas y negativas que influyen en el inicio y mantención de LME. Objetivo: Evaluar los efectos de las

Recibido el 06 de julio de 2012, devuelto para corregir el 04 de octubre de 2012, segunda versión el 05 de diciembre de 2012 , aceptado para publicación el 26 de enero de 2013.

Este trabajo cumple con los requisitos sobre consentimiento /asentimiento informado, comité de ética, financiamiento, estudios animales y sobre la ausencia de conflictos de intereses según corresponda.

Correspondencia a:

Camila Lucchini Raies

clucchin@uc.cl 
“co-variables" en la mantención de LME a los dos meses, según modalidad de atención. Pacientes y Método: Ensayo clínico aleatorio controlado con seguimiento de puérperas e hijos(as), respecto de prevalencia de LME a las $8 \mathrm{sem}$, y las co-variables positivas y negativas para LME en dos modalidades de asistencia de parto. Muestra: 649 puérperas (330 modalidad integral y 319 modalidad tradicional). Resultados: Prevalencia de LME a las 8 sem correspondió a $56,1 \%$ en modalidad integral y $48,9 \%$ en modalidad tradicional $(\mathrm{p}=0,06)$. Existen diferencias significativas en resultados de LME a las 8 sem según edad, paridad, escolaridad, entre ambas modalidades. Las "co-variables" positivas para LME se presentan en mayor proporción en modalidad integral $(\mathrm{p}<0,0001)$. Conclusión: Si bien, no existen variables que afecten de manera independiente el inicio y duración de LME a las $8 \mathrm{sem}$, la modalidad integral en su conjunto actuaría a su favor.

(Palabras clave: Lactancia materna exclusiva, prevalencia, atención integral del parto).

Rev Chil Pediatr 2013; 84 (2): 138-144

\section{Introducción}

La lactancia materna (LM) es la mejor alternativa para alimentar a los recién nacidos y lactantes, reportándose cada día más beneficios para el niño(a), la madre, su familia y la sociedad $^{1,2}$. En Chile, esta práctica ha sido una de las prioridades sanitarias desde 1990, razón por la cual se han implementado estrategias destinadas a su promoción, siendo la política "Chile Crece Contigo", la más reciente 3 .

El Servicio de Salud Metropolitano Sur Oriente (SSMSO) en conjunto con la Pontificia Universidad Católica de Chile, a partir de 2010 implementó una modalidad de atención integral del parto, en el Servicio de Maternidad del Hospital Sótero del Río (Proyecto FONDEF $N^{\circ}$ D07I1046). En dicho establecimiento en el año 2009 , un $79,4 \%$ de los nacidos vivos se alimentaba con lactancia materna exclusiva (LME) al mes de vida y un $67,3 \%$ lo hacía a los 3 meses de nacidos ${ }^{4}$. Cifras muy similares a las nacionales para las mismas edades y fecha 5 .

La OMS y UNICEF recomiendan el contacto piel a piel, el inicio precoz del amamantamiento y el alojamiento conjunto madrehijo(a) como estrategias para fomentar la atención integral del parto, favoreciendo el inicio, establecimiento y mantención de la $\mathrm{LME}^{6-9}$.

Existen prácticas de conducción del trabajo de parto que podrían obstaculizar el inicio precoz de la LM: uso de anestesia peridural, aceleración oxitócica, ruptura artificial de membranas (RAM), malestar materno, entre otros $^{10}$.

Dado que las variables anteriores se rela- cionan con el inicio y mantención de la lactancia durante los primeros meses postparto ${ }^{7-9}$, el objetivo del estudio es evaluar sus efectos en la mantención de la LME a los dos meses, según modalidad de atención del parto (integral y tradicional). La hipótesis es que la modalidad integral presentará una mayor tasa de lactancia materna a los 2 meses postparto.

\section{Pacientes y Método}

\section{Tipo de estudio y sujetos de investigación}

El universo de mujeres en estudio corresponde a embarazadas de bajo riesgo obstétrico, excluyendo a aquellas con patologías o factores de riesgo que requieran una vigilancia materna y/o perinatal más intensiva durante este proceso; con embarazo deseado y de gestación única, atendidas en el Hospital Dr. Sótero del Río, Santiago Chile (tabla 1).

Ensayo clínico aleatorio controlado que

\section{Tabla 1. Criterios de bajo riesgo obstétrico para el ingreso}

1. Embarazada sin patologías o factores de riesgo que requieran de vigilancia materna y/o perinatal más intensiva durante el proceso de trabajo de parto y parto

2. Inicio espontáneo del trabajo de parto:

a. Dinámica uterina espontánea de 2 a 3 en 10 min

b. Dilatación cervical mayor a $3 \mathrm{~cm}$ y más de $50 \%$ de borramiento del cuello uterino

3. Edad gestacional entre $37+0$ y $41+0$ sem

4. Embarazo único

5. Feto vivo

6. Presentación cefálica 
consideró el seguimiento de las puérperas y sus hijos(as), respecto de la prevalencia de LME a las 8 sem, y las co-variables positivas (contacto piel a piel, inicio precoz del amamantamiento y alojamiento conjunto madrehijo) y negativas para LME (anestesia peridural, aceleración oxitócica, ruptura artificial de membranas, malestar materno) en dos modalidades de asistencia de parto.

Las embarazadas fueron asignadas en forma aleatoria a cada modalidad de intervención, mediante un diseño en bloques aleatorios de 6 a 8 mujeres, de manera que en cada bloque se asignó igual número de mujeres a cada grupo. Esto permitió tener un número similar de embarazadas asignadas a cada modalidad durante todo el período de enrolamiento.

\section{Intervenciones}

La intervención en el grupo de asistencia integral consideró una modalidad menos intervencionista, con un porcentaje bajo de medidas de conducción (co-variables negativas para LME). La participación familiar fue permanente y activa durante toda la hospitalización, comprendiendo acompañamiento continuo de familiar significativo desde el ingreso hasta el alta, las $24 \mathrm{~h}$ del día, e involucrado en actividades de apoyo a la mujer durante todo el proceso. El parto se llevó a cabo en una sala integral de asistencia continua, donde se realizó contacto precoz piel con piel de al menos una hora y se promovió el inicio precoz de la LM (co-variables positivas para LME). Durante el postparto inmediato se desarrolló una intervención de apoyo educativa personalizada de responsabilidad del equipo de salud. El alta precoz en la modalidad integral (a partir de las $24 \mathrm{~h}$ ), se complementó con una visita domiciliaria (a partir de las $48 \mathrm{~h}$ ), donde se reforzaron los cuidados de la madre y el hijo (a), y la técnica de LM en su entorno familiar.

La modalidad tradicional correspondió a la atención habitual del sistema público que considera intervenciones de conducción del trabajo de parto (co-variables negativas para LME), con una participación familiar intermitente y pasiva. El contacto precoz piel a piel se realiza sin un protocolo estandarizado y el alojamiento conjunto se inicia una vez que el recién nacido ha recibido la atención inmediata. Durante el posparto en la unidad de puerperio se realiza apoyo profesional y técnico para el inicio de la LM. El alta, a partir de las 48 h, no considera visita domiciliaria como apoyo complementario a la intervención.

En ambas modalidades se realizó un seguimiento telefónico a las 8,16 y 24 sem postparto a todas las participantes, en donde se consultó respecto del tipo de alimentación que estaba recibiendo el niño (a). En este artículo se presentan los resultados obtenidos en el control de las 8 sem.

\section{Aspectos éticos y consentimiento}

Se contó con la aprobación de los Comités de Ética de la Escuela de Enfermería UC y del SSMSO. La muestra se constituyó por embarazadas que cumplieron los criterios de bajo riesgo obstétrico al momento del ingreso, que aceptaron participar en forma voluntaria, firmando el Consentimiento Informado.

\section{Análisis estadístico}

El cálculo del tamaño muestral se hizo en base a dos variables dependientes: la tasa de incidencia de cesárea y la prevalencia de lactancia materna exclusiva a los 6 meses. Estos cálculos se realizaron de modo de encontrar diferencias con significancia $\alpha=5 \%$ y potencia $1-\beta=80 \%$, asumiendo que se encontraría alrededor de $15 \%$ de cesáreas y un $55 \%$ de LME en la modalidad tradicional y un $11 \%$ de cesáreas y $63 \%$ de LME en la modalidad integral. Las variables numéricas fueron descritas en términos de promedio y desviación estándar, y las categóricas se presentan como número de casos y porcentaje. Se usó test $\chi^{2}$ de Pearson para comparar porcentajes y test $t$ de Student para muestras independientes para comparación de promedios. Para determinar la asociación conjunta de variables con LME, se ajustaron modelos de regresión logística binaria. Estos modelos se usaron también para controlar el efecto de eventuales variables confundentes en la relación de LME con otras variables de interés. Se consideró significativo todo valor $p \leq 0,05$. Los análisis se hicieron con el programa SAS 9.1 para Windows. 


\section{Resultados}

Hasta octubre de 2011, 770 embarazadas en trabajo de parto fueron asignadas en forma aleatoria al grupo intervención (modalidad integral) y al grupo control (modalidad tradicional). La muestra alcanzada para el control telefónico de las 8 sem correspondió a 649 puérperas (330 modalidad integral y 319 modalidad tradicional). La cantidad de casos perdidos $(15,7 \%)$ correspondió principalmente a cambio de número telefónico $\mathrm{y}$ de dirección (figura 1). Cabe destacar, que tanto las mujeres a las que se les realizó el control telefónico a las 8 sem como a las que no, eran similares respecto a su distribución entre grupos de estudio, en edad y según tipo de parto; así como también según peso, talla y edad gestacional de los recién nacidos, por lo que se espera que las pérdidas hayan sido al azar ("missing at random") (tabla 2).

El perfil bio-sociodemográfico de las puérperas según modalidad de atención del parto se detalla en la tabla 3. Cabe destacar que no existieron diferencias significativas entre ambas modalidades en las variables sociodemográficas y biológicas. La prevalencia de LME a las 8 sem correspondió a $56,1 \%$ en modalidad integral, y un $48,9 \%$ en modalidad tradicional $(\mathrm{p}=0,06)$.

Al analizar los resultados de LM por las variables bio-sociodemográficas separadas por modalidad, se observa que el grupo tradicional aumenta el porcentaje de LME a mayor edad de la madre $(\mathrm{p}=0,01)$, a diferencia de la modalidad integral donde no hay variaciones significativas para la variable edad $(\mathrm{p}=0,3)$. Al comparar los resultados de LM según rango etario, se observa que en el rango $<19$ años, el 56,3\% de las mujeres en el grupo integral se encuentran con LME a las 8 sem, versus el 39,1\% del grupo tradicional $(\mathrm{p}=0,03)$. Al analizar la escolaridad de la madre, se observa que en el grupo tradicional la LME a las 8 sem postparto es mayor a medida que el nivel
Tabla 2. Comparación entre seguimiento y pérdida

\begin{tabular}{|c|c|c|c|}
\hline Seguimiento & $\begin{array}{c}\text { Sí } \\
\text { Media }\end{array}$ & $\begin{array}{c}\text { No } \\
\text { Media }\end{array}$ & Valor $\mathbf{p}$ \\
\hline Edad puérpera & 23,9 & 24,4 & 0,37 \\
\hline Peso RN & 3.430 & 3.428 & 0,97 \\
\hline Talla RN & 49,7 & 49,9 & 0,89 \\
\hline \multirow[t]{2}{*}{ Edad gestacional } & 39,9 & 39,9 & 0,95 \\
\hline & $\%$ mujeres & $\%$ mujeres & Valor $\mathbf{p}$ \\
\hline Tipo de parto & & & 0,24 \\
\hline Vaginal sin episiotomía & 68,98 & 71,94 & \\
\hline Vaginal con episiotomía & 22,71 & 17,07 & \\
\hline Fórceps & 3,29 & 4,78 & \\
\hline Cesárea & 4,82 & 6,71 & \\
\hline
\end{tabular}

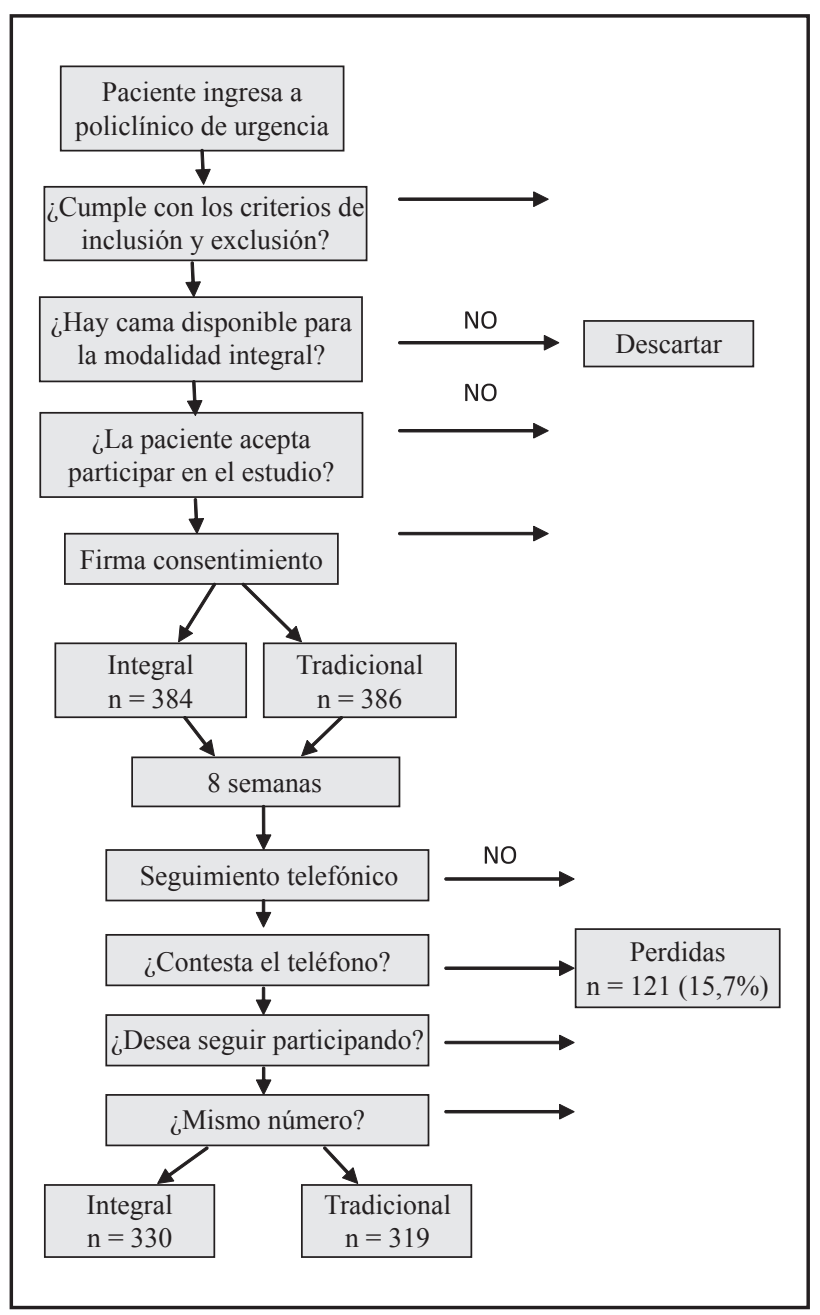

Figura 1. Flujograma ingreso de participantes a ambas modalidades. 
Tabla 3. Perfil biosociodemográfico según modalidad de atención

\begin{tabular}{|c|c|c|c|c|c|}
\hline Modalidad & Int & gral & Trad & cional & \\
\hline & & $\%$ & $\mathbf{n}$ & $\%$ & Valor $p$ \\
\hline Edad & & & & & 0,08 \\
\hline$\leq 19$ años & 71 & 21,5 & 87 & 27,3 & \\
\hline 20-30 años & 210 & 63,6 & 179 & 56,1 & \\
\hline$>31$ años & 49 & 14,9 & 53 & 16,6 & \\
\hline Paridad & & & & & 0,48 \\
\hline Primíparas & 131 & 39,7 & 140 & 44,3 & \\
\hline Multíparas & 199 & 60,3 & 176 & 55,7 & \\
\hline Escolaridad & & & & & 0,26 \\
\hline E. Básica & 62 & 18,8 & 50 & 15,7 & \\
\hline E. Media & 251 & 76 & 242 & 75,8 & \\
\hline E. Superior & 17 & 5,2 & 27 & 8,5 & \\
\hline Actividad materna & & & & & 0,08 \\
\hline Trabajo estable $\geq$ a $22 \mathrm{~h}$ & 53 & 16,1 & 60 & 18,8 & \\
\hline Trabajo esporádico & 12 & 3,6 & 13 & 4,1 & \\
\hline Sin actividad & 265 & 80,3 & 246 & 77,1 & \\
\hline APGAR familiar* & & & & & 0,78 \\
\hline $0-6$ & 28 & 8,5 & 29 & 9,1 & \\
\hline $7-10$ & 302 & 91,5 & 290 & 90,91 & \\
\hline Situación de pareja & & & & & 0,16 \\
\hline Pareja estable & 220 & 66,7 & 196 & 61,4 & \\
\hline Sin pareja estable & 110 & 33,3 & 123 & 38,6 & \\
\hline
\end{tabular}

*APGAR: Adaptabilidad, participación, crecimiento, afectividad y resolución. Cada miembro de la familia indica su grado de satisfacción puntuando de 0 a 2 cada categoría. El instrumento APGAR familiar permite evaluar el funcionamiento sistemático de la familia, considerándose útil en la identificación de familias en riesgo.

Tabla 4. Tipo de alimentación por modalidad según variables biosociodemográficas

\begin{tabular}{|lccc|}
\hline Modalidad & $\begin{array}{c}\text { Integral } \\
\text { \% LME }\end{array}$ & $\begin{array}{c}\text { Tradicional } \\
\text { \% LME }\end{array}$ & Valor $\mathbf{p}$ \\
Edad & & & \\
$\leq 19$ años & 56,34 & 39,08 & 0,03 \\
$20-30$ años & 53,81 & 49,16 & 0,36 \\
$>31$ años & 65,31 & 64,15 & 0,90 \\
Valor p total & 0,34 & 0,01 & \\
Paridad & & & \\
Primíparas & 50,38 & 49,62 & \\
Multíparas & 59,32 & 50 & \\
Valor $p$ total & 0,09 & 0,52 & \\
Escolaridad & & & \\
E. Básica & 53,23 & 38 & 0,10 \\
E. Media & 56,18 & 48,76 & 0,09 \\
E. Superior & 64,41 & 70,37 & 0,69 \\
Valor $p$ total & 0,79 & 0,02 & \\
Situación de pareja & & & \\
Pareja estable & 55 & 50 & \\
Sin pareja estable & 58,18 & 47,15 & \\
Valor $p$ total & 0,58 & 0,58 & \\
\hline
\end{tabular}

educacional aumenta $(\mathrm{p}=0,02)$, situación que no se presenta en la modalidad integral, en donde esta diferencia no es significativa $(\mathrm{p}=0,6)($ tabla 4$)$.

Al estudiar la variable paridad se observa que en las mujeres con un solo hijo anterior, la LME a las 8 sem fue de $63,3 \%$ para aquellas en la modalidad integral versus un $47,1 \%$ en la modalidad tradicional, diferencia estadísticamente significativa $(p=0,01)$. Para las otras variables estudiadas, no existen diferencias significativas en LME a las 8 sem, entre ambas modalidades de atención (tabla 4).

En relación a las "co-variables positivas" para LME (contacto precoz piel a piel e inicio de LM antes de las $2 \mathrm{~h}$ de vida), estas se presentan en mayor proporción en la modalidad integral, lo que es estadísticamente significativo $(\mathrm{p}<0,0001)$. La variable "alojamiento conjunto", no fue considerada, puesto que ambas modalidades la incorporan. Lo mismo sucedió, pero en la relación inversa, con las covariables negativas para LME (anestesia peridural, aceleración oxitócica, RAM y malestar materno), en donde se presentaron con mayor proporción en las mujeres pertenecientes a la modalidad tradicional $(\mathrm{p}<0,0001)$ (tabla 5).

Si bien, al comparar la modalidad tradicional versus modalidad asistencia integral no se observaron diferencias significativas, incluso después de ajustar por cada determinante de LME, al interior de cada grupo sí se observan patrones de comportamiento que muestran el beneficio de la modalidad integral.

\section{Discusión}

El presente trabajo demuestra una diferencia en la proporción de mujeres con LME a las 8 sem postparto según modalidad de atención, a favor de aquellas que tuvieron sus partos en la modalidad integral. Si bien es cierto, que esta diferencia no logra ser estadísticamente significativa, se aproxima a ella y da luces respecto a que una modalidad que 
Tabla 5. Presencia de co-variables positivas y negativas según modalidad de atención

\begin{tabular}{|lccccc|}
\hline Modalidad & \multicolumn{2}{c}{ Integral } & \multicolumn{2}{c}{ Tradicional } & Valor p \\
Contacto piel con piel $(+1$ h) & n & \% & n & \% & \\
Lactancia materna precoz antes de las $(2$ h) & 230 & 77,18 & 18 & 6,69 & $<0,0001$ \\
Aceleración oxitócica & 252 & 70,19 & 107 & 33,65 & $<0,0001$ \\
Rotura artificial de membrana (RAM) & 59 & 17,88 & 149 & 46,71 & $<0,0001$ \\
Uso de anestesia & 109 & 33,03 & 207 & 64,89 & $<0,0001$ \\
Bienestar materno & 169 & 51,2 & 271 & 84,95 & $<0,0001$ \\
Óptimo & & & & & \\
Malestar & 301 & 92,62 & 79 & 25,08 & $<0,0001$ \\
\hline
\end{tabular}

considera variables favorecedoras de la LM tiene efectos positivos en el establecimiento de ésta ${ }^{7,8,11}$.

Asimismo, destaca que en el grupo tradicional tanto la variable "edad" como "educación materna" afectan significativamente la proporción de LME a las ocho semanas postparto. No así, en el grupo de atención integral, en donde no existen diferencias significativas entre estas variables y la LME a las $8 \mathrm{sem}$. Estos resultados son avalados por la literatura disponible, que señala que tanto la edad como el nivel educacional de la madre son factores personales que influyen directamente en la duración de esta práctica ${ }^{12}$. Además, se encontró una relación estadísticamente significativa entre la variable "edad de la madre" y la mantención de la LME, para las mujeres de la modalidad integral $(p=0,03)$, en donde las menores de 19 años presentan una proporción mayor de LME que las del mismo grupo etario, pertenecientes a la modalidad tradicional. Se reporta que el grupo de madres adolescentes se caracteriza por una alta vulnerabilidad y necesidad de apoyo extra en el proceso de lactancia materna ${ }^{13}$. La modalidad integral de atención de parto considera apoyo permanente en el inicio de la LM, razón que podría explicar dicha diferencia.

Con respecto a la mayor proporción de LME en las mujeres con un hijo (a) anterior en la modalidad integral, no se encontró evidencia que avale dicho resultado. Sin embargo, sí existen publicaciones en donde la paridad de la madre se relaciona directamente con el aumento en la duración de la $\mathrm{LME}^{9,12}$.
Es notorio que existiendo diferencias significativas en la presencia de las co-variables positivas y negativas según modalidad, éstas no tengan relación directa con los resultados en la LME a las 8 sem de vida. Esto se contrapone con los resultados de investigaciones anteriores, en donde éstas "co-variables" sí impactan en los resultados de $\mathrm{LME}^{7,8,10}$. Sin embargo, estas investigaciones han considerado el estudio de las "co-variables" por separado o asociadas según efecto (positivo o negativo para LME) y no en su conjunto.

En conclusión, este trabajo nos permite señalar, que si bien es cierto, no existen variables que afecten de manera independiente el inicio y la duración de la LME a las 8 sem, al parecer la modalidad integral en su conjunto actuaría en su favor. Asimismo, esta modalidad integral permitiría disminuir las brechas de equidad existentes en la población de riesgo (según edad y escolaridad) para LME a las 8 sem postparto. Por último, cabe señalar que entre las limitaciones de este estudio se debe considerar, el porcentaje de pérdida en el seguimiento telefónico postparto, la falta de registro del tipo de alimentación recibida durante la hospitalización y al alta del recién nacido, información que habría permitido tener una visión más completa del comportamiento de las variables estudiadas.

\section{Referencias}

1.- Kramer M, Guo T, Platt R, et al: Infant growth and health outcomes associated with 3 compared with 6 mo of exclusive breastfeeding. Am J Clin Nutr [internet] 
2003 [Cited 2011 Dec 26]; 78 (2): 291-5. Available from: http://www.ajen.org/content/78/2/291.long

2.- Duijits L, Vincent J, Hofman A, Moll H: Prolonged and Exclusive Breastfeeding Reduces the Risks of Infectious Diseases in Infancy. Pediatrics 2010; 126 (1): e18-25. Doi:10.1542/peds.2008-3256.

3.- Chile crece contigo: Creciendo juntos: [interntet] Santiago: Chile. 2010 [Update 2011 Dec 12; cited 2011 Dec 26] Avaliable from: http://www.crececontigo.gob. cl/especialistas/documentos/

4.- DEIS MINSAL: Atenciones en maternidad. [Internet] 2011 [Update 2009 Dec 31; cited 2011 Dec 26]. Available from: http://intradeis.minsal.cl/Intradeis/Reportes_2009/PARTOS_CESAREA/PARTOS_CESAREA. aspx

5.- DEIS MINSAL: Controles realizados al tercer mes según tipo de lactancia. [Internet] 2011 [Update 2009 Dec 31; cited 2012 Jun 18]. Available from: http:// intradeis.minsal.cl/Intradeis/Reportes_2009/LACTANCIA_MATERNA_3M/LACTANCIA_MATERNA_3M. aspx

6.- Hodnett E, Gates S, Hofmeyr G, Sakala: Continuous support for women during childbirth. Cochrane Databse of Systematic Revies [Internet] 2007[Cited 2011 Dec 26] Available from: http://apps.who.int/rhl/pregnancy_chil$\mathrm{dbirth/childbirth/routine \_ care/hebcom/es/}$

7.- Bramson L, Lee J, Moore E, et al: Effect of Early Skinto-Skin Mother-Infant Contact During the First 3 hours Following Birth on Exclusive Breastfeeding During the Maternity Hospital Stay. J hum lact 2010; 26 (2): 131-7.
Doi: $10.1177 / 0890334409355779$

8.- DiGirolamo A, Grummer-Strauwn L, Fein S: Effect of Maternity-Care Practices on Breastfeeding. Pediatrics. 2008; 122 (Suppl 2) S43-9: doi:10.1542/peds.2008$1315 \mathrm{e}$

9.- Bystrova K, Widström A, Matthiesen A, et al: Early lactation perfomance in primiparous and multiparous women in relation to different maternity home practices, A randomised trial in St.Petersburg. Int breastfeed $\mathrm{j}$. 2007 May; 2 (9): 9-14. Doi: 10.1186/1746-4358-2-9.

10.- Gizzo S, Di Gangi S, Saccardi C, et al: Epidural Analgesia During Labor: Impact on Delivery Outcome, Neonatal Well-Being, And Eraly Breastfeeding. Breastfeeding Med. 2011. 13.Doi:10.1089/bfm.2011.0099

11.- Romero M, Algaba S, Albar M, Núñez E, Calero C, Pérez I: Influencia de las prácticas hospitalarias en el inicio y mantenimiento de la lactancia materna. Enferm clin. [Internet] 2004 [Cited 2012 Jun 18]; 14 (4): 194202. Available from: http://www.elsevier.es/es/abstract/ revistas/enfermeria-clinica-35/influencia-las-practicashospitalarias-inicio-mantenimiento-lactancia-13065572originales-2004?bd=1

12.- Hauck Y, Fenwinck J, Dhaliwal S, Butt J: A Western Australian Survey of Breastfeeding Initiation Prevalence and Early Cessation Patterns. Matern Child Health J. 2011; 15: 260-268.Doi: 10.1007/s10995-009-0554-2.

13.- Mossamn M, Heaman M, Dennis C, Morris M: The Influence of Adolescent Mothers' Breastfeeding Confidence and attitudes on Breastfeeding initiation. J Hum Lact 2008.24: 268. Doi: 10.1177/089033408316075. 